\title{
PHASE IN OPTICS
}

ฮี

包

.气.

o

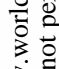

3

全

ह

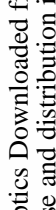

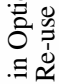

0

종

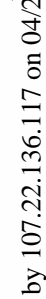




\section{SERIES IN CONTEMPORARY CHEMICAL PHYSICS}

Editor-in-Chief: M. W. Evans (York University, Toronto)

Associate Editors: S Jeffers (York University, Toronto)

D Leporini (University of Pisa, Italy)

J Moscicki (Japiellonian University, Poland)

L Pozhar (The Ukrainian Academy of Sciences)

S Roy (The Indian Statistical Institute)

Vol. 1 The Photon's Magnetic Field - Optical NMR Spectroscopy by M. W. Evans and F. Fahari

Vol. 2 Beltrami Fields in Chiral Media by A. Lakhtakia

Vol. 3 Quantum Mechanical Irreversibility and Measurement by P. Grigolini

Vol. 4 The Photomagneton and Quantum Field Theory: Quantum Chemistry, Vol. 1 by A. A. Hasanein and M. W. Evans

Vol. 5 Computational Methods in Quantum Chemistry: Quantum Chemistry, Vol. 2 by A. A. Hasanein and M. W. Evans

Vol. 6 Transport Theory of Inhomogeneous Fluids by L. A. Pozhar

Vol. 7 Dynamic Kerr Effect: The Use and Limits of the Smoluchowski Equation and Nonlinear Inertial Responses by J.-L. Dejardin

Vol. 8 Dielectric Relaxation and Dynamics of Polar Molecules by V. I. Gaiduk (forthcoming)

Vol. 9 Water in Biology, Chemistry and Physics: Experimental Overviews and Computational Methodologies

by G. W. Robinson, S. B. Zhu, S. Singh and M. W. Evans

Vol. 10 The Langevin Equation: With Applications in Physics, Chemistry and Electrical Engineering by W. T. Coffey, Yu P. Kalmykov and J. T. Waldron

Vol. 11 Structure and Properties in Organised Polymeric Materials eds. E. Chiellini, M. Giordano and D. Leporini

Vol. 12 Proceedings of the Euroconference on Non-Equilibrium Phenomena in Supercooled Fluids, Glasses and Amorphous Materials eds. M. Giordano, D. Leporini and M. P. Tosi

Vol. 13 Electronic Structure and Chemical Bonding by J. R. Lalanne

Vol. 14 Dialogues on Modern Physics by $M$. Sachs 


\section{PHASE IN OPTICS}

\section{Vlasta Peřinová, Antonín Lukš and Jan Peřina}

Palacký University, Olomouc, Czech Republic 


\section{Published by}

World Scientific Publishing Co. Pte. Ltd.

P O Box 128, Farrer Road, Singapore 912805

USA office: Suite 1B, 1060 Main Street, River Edge, NJ 07661

UK office: 57 Shelton Street, Covent Garden, London WC2H 9HE

\section{Library of Congress Cataloging-in-Publication Data}

Peřnová, Vlasta, 1943-

Phase in optics / Vlasta Perinová, Antonín Lukš, Jan Perina.

p. cm. -- (World Scientific series in contemporary chemical physics ; vol. 15)

ISBN $981023208 \mathrm{X}$

1. Quantum optics. 2. Optical phase conjugation. 3. Phase space (Statistical physics) I. Lukš, Antonín, 1944- II. Perina, Jan, 1936- . III. Title. IV. Series.

QC446.2.P465 1998

535--dc21

\section{British Library Cataloguing-in-Publication Data}

A catalogue record for this book is available from the British Library.

Copyright $\odot 1998$ by World Scientific Publishing Co. Pte. Ltd.

All rights reserved. This book, or parts thereof, may not be reproduced in any form or by any means, electronic or mechanical, including photocopying, recording or any information storage and retrieval system now known or to be invented, without written permission from the Publisher.

For photocopying of material in this volume, please pay a copying fee through the Copyright Clearance Center, Inc., 222 Rosewood Drive, Danvers, MA 01923, USA. In this case permission to photocopy is not required from the publisher.

This book is printed on acid-free paper. 


\section{Preface}

The knowledge about the wave nature of particles, as a seed of particle optics, was one of the sources of quantum theory and introduced the concept of phase factors. At high speed optics improved our understanding and use of the wave nature of light in the classical regime.

But quantum field theory as a second-quantized theory has been plagued with problems of promotion of the classical phase to the quantum one since the beginning. In this theory the quantum phase could be expected only where the harmonic oscillator would reappear.

When quantum optics came into being, the radiation modes began to be studied as quantum harmonic oscillators and the hopes started to come true. But not without corrections.

The purpose of this book is to discuss these questions. To pursue this goal we pay great attention to the optical phase in classical regime, and also present information about particle optics and about the geometric (Berry and Pancharatnam) phases. We expound the coherent state technique, which has been useful for founding quantum optics. The most important part of this book discusses typical problems of the phase of a quantum harmonic oscillator and thus those of an optical mode. We take into account the contemporary literature concerning the simultaneous measurement of the position-like and momentum-like field quadratures and the reconstruction of a quantum state.

This book may be considered a continuation of previous monographs by one of the authors on Coherence of Light (Van Nostrand Reinhold, London 1972, second edition D. Reidel, Dordrecht 1985) and on Quantum Statistics of Linear and Nonlinear Optical Phenomena (D. Reidel, Dordrecht 1984; second edition Kluwer, Dordrecht 1991), and on Quantum Optics and Fundamentals of Physics (Kluwer, Dordrecht 1994) co-authored by Z. Hradil and B. Jurčo, which also cover the prerequisites for reading this book.

The book will be useful to research workers in general optics, quantum optics and electronics, optoelectronics, and nonlinear optics, as well as to students of physics, optics, optoelectronics, photonics, and optical engineering.

The text of the book has been worked out as follows: Chapters 2, 3, and 5 have been written by J. Per̆ina except sections 3.1, 3.9, 3.10, 5.3, and 5.4. All the other 
text is by V. Peřinová and A. Lukš.

We would like to thank Mgr. R. Böhm for carefully typing a part of the manuscript. We are obliged to Dr. Jan Peřina Jr. and Ing. J. Křepelka for careful preparation of figures. We express our gratitude to Professors Emil Wolf and Malvin Carl Teich $\dot{s}$ for their kind help and deep interest in this work. V. P. and A. L. acknowledge the Grant Agency of Czech Republic for supporting the investigation of quantum optical interpretation of phase of the light wave and of the use of quantum phase in the analysis of optical processes and optics measurements by grant 202/93/0011. J. P. would like to thank the Grant Agency of Czech Republic and Czech Ministry of Education ळ for supports of this research by grants 202/96/0421 and VS96028, respectively.

Olomouc, December 1997

\author{
Vlasta Peřinová \\ Antonín Lukš \\ Jan Peřina
}




\section{Contents}

Preface

1 Introduction 1

2 Phase in classical and nonlinear optics 5

2.1 Interference of light . . . . . . . . . . . . . . . . . 5

2.2 Diffraction of light . . . . . . . . . . . . . . . . . . . 12

2.3 Holography . . . . . . . . . . . . . . . . . . . . . . . . . . 20

2.4 Phase conjugation . . . . . . . . . . . . . . . . . . . 23

2.5 Phase retrieval methods . . . . . . . . . . . . . 25

3 Phase-space description of light field 33

3.1 Wigner function for two arbitrary operators . . . . . . . . . . . 33

3.1 .1 Operator-valued measures . . . . . . . . . . . . 34

3.1.2 Generalized Wigner function as a measure . . . . . . . . . 39

3.2 Basic properties of coherent states . . . . . . . . . . . . . 44

3.3 Quantum characteristic functions and quasidistributions . . . . . . 49

3.4 Operator orderings . . . . . . . . . . . . . . . . 56

3.5 Squeezed states . . . . . . . . . . . . . . . . . . . . 6 66 66

3.6 Atomic coherent states . . . . . . . . . . . . . . . . . 72

3.7 Photocount statistics . . . . . . . . . . . . . . . . . . . 75

3.8 Generalized superposition of coherent fields and quantum noise . . . 77

3.9 Irreversible quantum dynamics . . . . . . . . . . . . . . 80

3.10 Generation and decay of superposition states . . . . . . . . 100

4 Phase in quantum optics 117

4.1 Phase and the "old" quantum theory . . . . . . . . . . . . . . . 119

4.2 Geometric phase . . . . . . . . . . . . . . . . . . 127

4.3 Quantum phase problem ... . . . . . . . . . . . 136

4.4 Statistical treatment of angle and phase, measures of phase variability 154

4.4.1 One random phase variable and two independent random phase variables . . . . . . . . . . . . . . . 156 
4.4.2 Two correlated random phase variables . . . . . . . . . . 167

4.5 Orderings of exponential phase operators . . . . . . . . . . . . . 176

4.5 .1 Analogy . . . . . . . . . . . . . . . . . . 176

4.5.2 Quasidistributions for quantum cosine and sine . . . . . . 182

4.6 Enlargement of the Hilbert space of a harmonic oscillator . . . . . . 186

4.6.1 Number-product vacuum states . . . . . . . . . . . . . . . . 187

4.6 .2 States of a plane rotator . . . . . . . . . . . . . . 189

4.6.3 The original and enlarged Hilbert spaces . . . . . . . . . . . 190

4.7 Restricted Hilbert space of a harmonic oscillator . . . . . . . . . . . 201

4.8 Quantum phase for multimode fields . . . . . . . . . . . . . . . . 224

4.8.1 Multimode formalisms of quantum phase . . . . . . . . . . 224

4.8.2 Grey is the phase sum and the phase difference is green . . . . 228

4.9 Number-phase extremal states . . . . . . . . . . . . . . . . . . . 235

4.9.1 Special states of the optical oscillator . . . . . . . . . . 236

4.9 .2 Special states of the plane rotator . . . . . . . . . . . 243

4.9.3 Special states from the truncated Hilbert space of optical oscillator . . . . . . . . . . . . . . . . . . 249

$4.9 .4 \mathrm{SU}(2)$ intelligent states . . . . . . . . . . . . . . . 252

$4.9 .5 \mathrm{SU}(1,1)$ intelligent states . . . . . . . . . . . . . . . . 253

4.10 Quantum phase and phase-space quasidistributions . . . . . . . 255

4.10 .1 Single-mode optical fields . . . . . . . . . . . . . . . . . 255

4.10 .2 Multimode optical fields . . . . . . . . . . . . . . . . . . 262

4.10.3 Quantum phase difference/sum and SU(2)/SU(1,1) phase-space quasidistributions . . . . . . . . . . . . 266

4.11 Phase properties of real states of optical field . . . . . . . . . . . . 266

4.11.1 Single-mode and two-mode superposition states . . . . . . . . 267

4.11.2 Single-mode and two-mode ordinary squeezed states . . . . . . 274

4.11 .3 Miscellaneous two-mode states . . . . . . . . . . . . . . . . . 284

4.12 Operational approach to quantum phase . . . . . . . . . . . . . 289

4.13 Concepts of ideal and feasible phases . . . . . . . . . . . . . 300

4.13 .1 Heterodyne detection of quadratures . . . . . . . . . . . . 304

4.13.2 Analogy between the feasible phase operator and the rotationangle operator . . . . . . . . . . . . . . . . . 308

4.13.3 Reconstruction of the statistical operator . . . . . . . . . 314

5 Phase-shift measurements and phase dependence 321

5.1 Quantum light interferometry . . . . . . . . . . . . . . . . 321

5.2 Nondemolition measurements . . . . . . . . . . . . . . . . . . . . 349

5.3 Phase-dependent measurements . . . . . . . . . . . . . . . 353

5.4 Phase dependence and phase dynamics in nonlinear optical processes 370

5.4 .1 Phase dependence . . . . . . . . . . . . . . . . 370

5.4 .2 Phase dynamics . . . . . . . . . . . . . . . . . 377 
5.4.3 Phase dynamics of $\ell$-photon coherent states in a Kerr medium 388 5.5 Particle interferometry . . . . . . . . . . . . . . . 401

6 Conclusion 441

$\begin{array}{ll}\cdot \text { References } & 415\end{array}$

$\begin{array}{ll}\text { Index } & 443\end{array}$

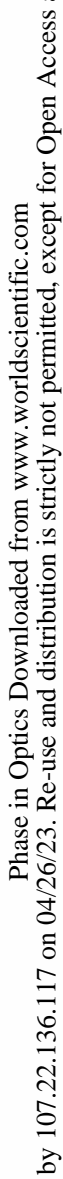

